\title{
On the complement of the Richardson orbit
}

\section{Journal Article}

Author(s):

Baur, Karin; Hille, Lutz

Publication date:

2012-10

Permanent link:

https://doi.org/10.3929/ethz-b-000044097

Rights / license:

In Copyright - Non-Commercial Use Permitted

Originally published in:

Mathematische Zeitschrift 272(1-2), https://doi.org/10.1007/s00209-011-0920-9 


\title{
On the complement of the Richardson orbit
}

\author{
Karin Baur • Lutz Hille
}

Received: 5 April 2010 / Accepted: 15 July 2011 / Published online: 10 August 2011

C) Springer-Verlag 2011

\begin{abstract}
We consider parabolic subgroups of a general linear group over an algebraically closed field $k$ whose Levi part has exactly $t$ factors. By a classical theorem of Richardson, the nilradical of a parabolic subgroup $P$ has an open dense $P$-orbit. In the complement to this dense orbit, there are infinitely many orbits as soon as the number $t$ of factors in the Levi part is $\geq 6$. In this paper, we describe the irreducible components of the complement. In particular, we show that there are at most $t-1$ irreducible components. We are also able to determine their codimensions.
\end{abstract}

Keywords Parabolic groups $\cdot$ Richardson orbit · Nilradical

Mathematics Subject Classification (2000) 20G05 - 17B45 · 14L35

\section{Contents}

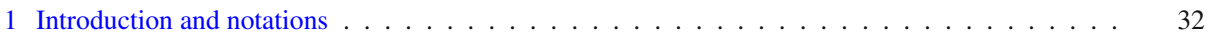

2 Components via rank conditions . . . . . . . . . . . . . . . . . . . . . . . . . . . . . . . . . . . . . . . . . .

2.1 Line diagrams . . . . . . . . . . . . . . . . . . . . . . . . . . . . . . . . . . . . . . . . . . . . . . . . .

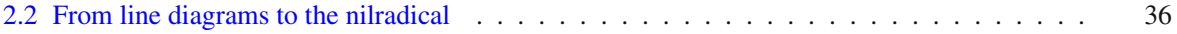

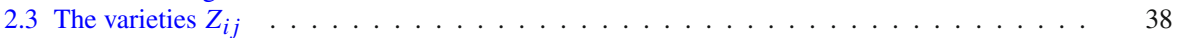

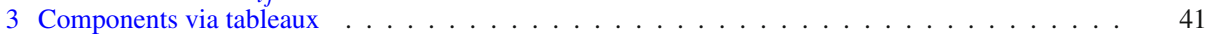

This research was supported through the programme "Research in Pairs" by the Mathematisches Forschungsinstitut Oberwolfach in 2009. K. Baur was supported by SNF. L. Hille was supported by the DFG priority program SPP 1388 representation theory.

K. Baur $(\bowtie)$

Department of Mathematics, ETH Zürich, Rämistrasse 101, 8092 Zurich, Switzerland

e-mail: baur@math.ethz.ch

L. Hille

Mathematisches Institut, Fachbereich Mathematik und Informatik der Universität Münster,

Einsteinstrasse 62, 48149 Münster, Germany

e-mail: lutz.hille@uni-muenster.de 


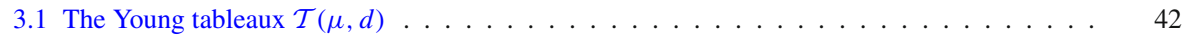

3.2 The Young tableaux $T(i, j) \ldots \ldots \ldots \ldots \ldots \ldots \ldots$

4 The irreducible components of $Z$. . . . . . . . . . . . . . . . . . . . . . . . . 45

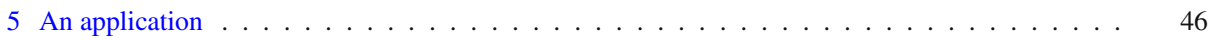

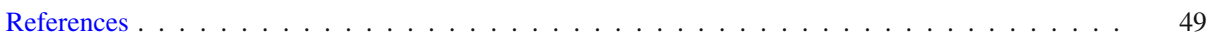

\section{Introduction and notations}

Let $P$ be a parabolic subgroup of a reductive algebraic group $G$ over an algebraically closed field $k$. Let $\mathfrak{p}$ be its Lie algebra and let $\mathfrak{p}=\mathfrak{l} \oplus \mathfrak{n}$ be the Levi decomposition of $\mathfrak{p}$, i.e. $\mathfrak{n}$ is the nilpotent radical of $\mathfrak{p}$. A classical result of Richardson [13] says that $P$ has an open dense orbit in the nilradical. We will call this $P$-orbit the Richardson orbit for $P$. However, in general there are infinitely many $P$-orbits in $\mathfrak{n}$.

For classical $G$, the cases where there are finitely many $P$-orbits in $\mathfrak{n}$ have been classified in [12]. Also, the $P$-action on the derived Lie algebras of $\mathfrak{n}$ have been studied in a series of papers, and the cases with finitely many orbits have been classified, cf. [3-5,7].

If $G$ is a general linear group, $G=\mathrm{GL}_{n}$, then the parabolic subgroup $P$ can be described by the lengths of the blocks in the Levi factor: write $P=L N$ where $L$ is a Levi factor and $N$ is the unipotent radical of $P$. Then we can assume that $L$ consists of matrices which have non-zero entries in square blocks on the diagonal. Similarly, the Levi factor $\mathfrak{l}$ of $\mathfrak{p}$ consists of the $n \times n$-matrices with non-zero entries lying in squares of size $d_{i} \times d_{i}(i=1, \ldots, t)$ on the diagonal and $\mathfrak{n}$ are the matrices which only have non-zero entries above and to the right of these square blocks.

Let $t$ be the number of such blocks and $d_{1}, \ldots, d_{t}$ the lengths of them, $\sum d_{i}=n$ (with $d_{i}>0$ for all $\left.i\right)$. So $d$ is a composition of $n$. We will call such a $d=\left(d_{1}, \ldots, d_{t}\right)$ a dimension vector. We write $P(d)$ for the corresponding parabolic subgroup and $\mathfrak{n}(d)$ for the nilpotent radical of $P(d)$, the Richardson orbit of $P(d)$ is denoted by $\mathcal{O}(d)$. Its partition will be $\lambda(d)$. Once $d$ is fixed, we will often just use $P, \mathfrak{n}$ and $\lambda$ if there is no ambiguity. Recall that the nilpotent $\mathrm{GL}_{n}$-orbits are parametrised by partitions of $n$. We will use $C(\mu)$ to denote the nilpotent $\mathrm{GL}_{n}$-orbit for the partition $\mu(\mu$ a partition of $n)$. And we will usually denote $P$-orbits in $\mathfrak{n}$ by a calligraphic $\mathrm{O}$, i.e. we will write $\mathcal{O}$ or $\mathcal{O}(\mu)$ if $\mu$ is the partition of the nilpotency class of the $P$-orbit.

Now, the nilradical $\mathfrak{n}$ is a disjoint union of the intersections $\mathfrak{n} \cap C(\mu)$ of the nilradical with all nilpotent $\mathrm{GL}_{n}$-orbits. By Richardsons result, $\mathfrak{n} \cap C(\lambda)=\mathcal{O}(\lambda)$ is a single $P$-orbit. In particular, the Richardson orbit consists exactly of the elements of the nilpotency class $\lambda$. However, for $\mu \leq \lambda$, the intersection $\mathfrak{n} \cap C(\mu)$ might be reducible (cf. Proposition 3.3).

In the case where $\mathfrak{n}$ is the nilradical of a Borel subalgebra of the Lie algebra of a simple algebraic group $G$, Spaltenstein has first studied the varieties $\mathfrak{n} \cap(G \cdot e)$ for $G \cdot e$ a nilpotent orbit under the adjoint action [14]. In [9], the authors study the action of a Borel subgroup $B$ of a simple algebraic group on the closure $\mathfrak{n} \cap C(\mu)$ for the subregular nilpotency class $C(\mu)$ and characterize the cases where $B$ has only finitely many orbits under the adjoint action.

The main goal of this article is to describe the irreducible components of the complement $Z:=\mathfrak{n} \backslash \mathcal{O}(d)$ of the Richardson orbit in $\mathfrak{n}$. They occur in intersections $\mathfrak{n} \cap C(\mu)$ for certain partitions $\mu=\mu(i, j) \leq \lambda$.

We have two descriptions of the irreducible components of $Z$. On one hand, we give rank conditions on the matrices of $\mathfrak{n}$, on the other hand, we use tableaux $T(i, j)$ for certain $(i, j)$ with $1 \leq i<j \leq t$ and associate irreducible components $\mathfrak{n}(T(i, j))$ of the intersections $\mathfrak{n} \cap C(\mu(i, j))$ to them. Before we can state the two results we now introduce the necessary notation. 
Fig. 1 The block decomposition of the matrix $A$ for $d=(2,4,7)$

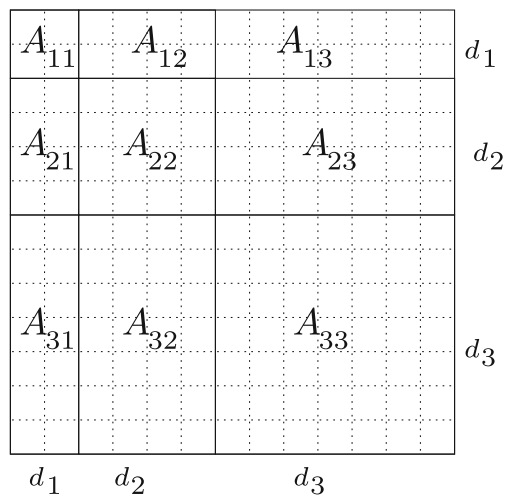

Let $d=\left(d_{1}, \ldots, d_{t}\right)$ be a dimension vector, $\mathfrak{n}$ the nilradical of the corresponding parabolic subalgebra. For $A \in \mathfrak{n}$ and $1 \leq i, j \leq t$ we write $A_{i j}$ to describe the matrix formed by taking the entries of $A$ lying in the rectangle formed by rows $d_{1}+\cdots+d_{i-1}+1$ up to $d_{1}+\cdots+d_{i}$ and columns $d_{1}+\cdots+d_{j-1}+1$ up to $d_{1}+\cdots+d_{j}$ and with zeroes everywhere else. For $i \geq j$, this is just the zero matrix. Figure 1 shows the blocks $A_{i j}$ for $d=(2,4,7)$.

We set $A[i, j]$ to be the matrix formed by the $\left(A_{k l}\right)_{i \leq k \leq j, i \leq l \leq j}$, i.e. by the rectangles right to and below of $A_{i i}$ and left to and above of $A_{j j}$. For instance, $A[i, i]$ is just $A_{i i}$ and $A[1, t]$ has the same entries as $A$. More generally, $A[i j]$ is a square matrix of size $\left(d_{i}+\cdots+d_{j}\right) \times\left(d_{i}+\cdots+d_{j}\right)$ with $A_{i i}, \ldots, A_{j j}$ on its diagonal.

We are now ready to explain the rank conditions. For the rest of this section, we will always assume that a pair $(i, j)$ satisfies $1 \leq i<j \leq t$. We write $X(d)$ for an element of $\mathcal{O}(d)$. For $k \geq 1$ define

$$
\begin{aligned}
r_{i j}^{k} & :=\operatorname{rk}\left(X(d)[i, j]^{k}\right) \\
\kappa(i, j) & :=1+\#\left\{l \mid i<l<j, d_{l} \geq \min \left(d_{i}, d_{j}\right)\right\} .
\end{aligned}
$$

Observe that the numbers $r_{i j}^{k}$ are independent of the choice of an element of the Richardson orbit. With this, we can define two subsets of $\mathfrak{n}$ as our candidates for irreducible components of $Z$.

Definition 1.1 Let $d=\left(d_{1}, \ldots, d_{t}\right)$ be a dimension vector and $\mathfrak{n}$ the nilradical of the parabolic subgroup $P$ of $\mathrm{GL}_{n}$. We set

$$
\begin{aligned}
& Z_{i j}^{k}:=\left\{A \in \mathfrak{n} \mid \operatorname{rk} A[i j]^{k}<r_{i j}^{k}\right\} \\
& Z_{i j}:=Z_{i j}^{\kappa(i, j)}
\end{aligned}
$$

to be the elements $A$ of $\mathfrak{n}$ for which the rank of $k$ th power of the matrix $A[i j]$ is defective, respectively, the $A$ for which the rank of the $\kappa(i, j)$ th power is defective.

To any dimension vector $d=\left(d_{1}, \ldots, d_{t}\right)$ we associate subsets $\Gamma(d)$ and $\Lambda(d)$ of the set $\{(i, j) \mid 1 \leq i<j \leq t\}$. In Sect. 2 we will show that the complement $Z$ of the open dense orbit is the union of the sets $Z_{i j}$ for $(i, j) \in \Lambda(d)$.

$$
\begin{aligned}
& \Gamma(d):=\left\{(i, j) \mid d_{l}<\min \left(d_{i}, d_{j}\right) \text { or } d_{l}>\max \left(d_{i}, d_{j}\right) \forall i<l<j\right\}, \\
& \Lambda(d):=\left\{(i, j) \in \Gamma(d) \mid d_{i}=d_{j}\right\} \cup
\end{aligned}
$$




$$
\left.\begin{array}{l}
\left\{(i, j) \in \Gamma(d) \mid d_{i} \neq d_{j}\right. \text { and } \\
\quad \text { (i) } d_{k} \leq \min \left(d_{i}, d_{j}\right) \text { or } d_{k} \geq \max \left(d_{i}, d_{j}\right) \forall k \\
\quad \text { (ii) } d_{k} \neq d_{j} \text { for } k<i \\
\text { (iii) } d_{k} \neq d_{i} \text { for } k>j
\end{array}\right\}
$$

Let us describe the latter in words: for $(i, j)$ to be in $\Lambda(d)$, we require that the $d_{l}$ with $i<l<j$ are smaller than the minimum of $d_{i}$ and $d_{j}$ or larger than the maximum of them. Furthermore, the $d_{k}$ have to be smaller or larger than the minimum $\min \left(d_{i}, d_{j}\right)$ resp. the maximum $\max \left(d_{i}, d_{j}\right)$ (for all $k$ ) and, if $d_{i} \neq d_{j}$, then $d_{i}$ is different from $d_{j+1}, \ldots, d_{t}$ and $d_{j}$ is different from $d_{1}, d_{2}, \ldots, d_{i-1}$. In general, $\Gamma(d)$ is different from $\Lambda(d)$ as we illustrate now.

Example 1.2 (a) If $d=(1,3,4,2)$ then $\Gamma(d)=\{(1,2),(2,3),(3,4),(2,4),(1,4)\}$ and $\Lambda(d)=\{(2,3),(2,4),(1,4)\}$.

(b) For $d=(1,2,3,2), \Gamma(d)=\{(1,2),(2,3),(3,4),(2,4)\}, \Lambda(d)=\{(1,2),(2,4)\}$.

(c) If $d=\left(d_{1}, \ldots, d_{t}\right)$ is increasing or decreasing, then $\Gamma(d)=\Lambda(d)=\{(1,2),(2,3), \ldots,(t-1, t)\}$.

(d) The fourth example will be our running example throughout the paper: if $d=(7,5,2,3,5,1,2,6,5)$ then we have $\Gamma(d)=\{(i, i+1) \mid 1 \leq i \leq 8\}$ $\cup\{(1,8),(2,4), \quad(2,5),(3,6),(3,7),(4,6),(4,7),(5,7),(5,8),(5,9),(7,9)\}$ and $\Lambda(d)=\{(1,8),(2,5),(3,7),(5,9)\}$.

We claim that the irreducible components of $Z=\mathfrak{n} \backslash \mathcal{O}(d)$ are the $Z_{i j}$ with $(i, j)$ from the parameter set $\Lambda(d)$ :

Theorem (Theorem 4.1) Let $d=\left(d_{1}, \ldots, d_{t}\right)$ be a composition of $n, \lambda=\lambda(d)$ the partition of the Richardson orbit corresponding to $d$. Then

$$
Z=\bigcup_{(i, j) \in \Lambda(d)} Z_{i j}
$$

is the decomposition of $Z$ into irreducible components.

For the second description of the irreducible components we let $T(d)$ be the unique Young tableau obtained by filling the Young diagram of $\lambda$ with $d_{1}$ ones, $d_{2}$ twos, etc. (for details, we refer to Sect. 3.1). Now for each pair $(i, j)$ we write $s(i, j)$ for the last row of $T(d)$ containing $i$ and $j$ and we let $T(i, j)$ be the tableau obtained from $T(d)$ by removing the box containing the number $j$ from row $s(i, j)$ and inserting it at the next possible position in order to obtain another tableau. The tableau $T(i, j)$ corresponds to an irreducible component of the intersection of $\mathfrak{n}$ with a nilpotent $\mathrm{GL}_{n}$-orbit as is explained in Sect. 3 (Proposition 3.3). We write $\mathfrak{n}(T(i, j)) \subseteq \mathfrak{n}$ for the irreducible component in $\mathfrak{n} \cap C(\mu(i, j)$ of tableau $T(i, j)$. We claim that they correspond to irreducible components of $Z$ exactly for the $(i, j) \in \Lambda(d)$.

Theorem (Corollary 4.4) Let $d=\left(d_{1}, \ldots, d_{t}\right)$ be a dimension vector, $\lambda=\lambda(d)$ the partition of the Richardson orbit corresponding to $d$. Then

$$
Z=\bigcup_{(i, j) \in \Lambda(d)} \mathfrak{n}(T(i, j))
$$

is the decomposition of $Z$ into irreducible components.

As a consequence, we obtain that $Z$ has at most $t-1$ irreducible components (cf. Corollary 4.2) and we can describe their codimensions in $\mathfrak{n}$ (Corollary 4.3). To be more precise, if $d$ is increasing or decreasing or if all the $d_{i}$ are different, then $Z$ has $t-1$ irreducible components. 
In particular, this applies to the Borel case where $d=(1, \ldots, 1)$. An example with $t=9$ and where we only have four irreducible components is our running example, see Example 3.8.

Note that the techniques we use are similar to the ones of [2] where we describe the complement to the generic orbit in a representation space of a directed quiver of type $\mathrm{A}_{t}$. However, the indexing sets are different and cannot be derived from each other.

The paper is organised as follows: in Sect. 2 we explain how to obtain the rank conditions. We first describe line diagrams associated to a composition $d$ of $n$. Line diagrams will be used to describe elements of the corresponding nilradical $\mathfrak{n}$. In Sect. 2.3 we prove that the elements of $\Lambda(d)$ give the irreducible components. For this, we show that if $(i, j)$ does not belong to $\Gamma(d)$ then the variety $Z_{i j}$ is contained in a union of $Z_{k_{s} l_{s}}$ for a subset of elements $\left(k_{s}, l_{s}\right)$ of $\Gamma(d)$ (Lemma 2.11). Next, if $(i, j)$ is in $\Gamma(d) \backslash \Lambda(d)$, then we can find $(k, l) \in \Lambda(d)$ such that $Z_{i j}$ is contained in $Z_{k l}$ (Corollary 2.13). In Sect. 3, we recall Young diagrams and their fillings. Then we consider Young tableaux associated to a composition $d$ of $n$ and a nilpotency class $\mu \leq \lambda(d)$. In a next step, we consider Young tableaux $T(i, j)$ associated to the elements of the parameter set $\Lambda(d)$. To each of these tableaux $T(i, j)$ we associate an irreducible variety $\mathfrak{n}(T(i, j))$ : it is defined as the irreducible component in $\mathfrak{n} \cap C(\mu(i, j))$ corresponding to the tableau $T(i, j)$. The $\mathfrak{n}(T(i, j))$ are known to be irreducible by work of the second author [10]. By showing that $\mathfrak{n}(T(i, j))$ is equal to $Z_{i j}$ from Sect. 2 for elements $(i, j)$ of the parameter set $\Lambda(d)$ we can complete the description of the complement of the Richardson orbit in $\mathfrak{n}$ into irreducible components.

\section{Components via rank conditions}

\subsection{Line diagrams}

Let $d=\left(d_{1}, \ldots, d_{t}\right)$ be a dimension vector for a parabolic subalgebra of $\mathfrak{g l}_{n}, \mathfrak{n}$ the corresponding nilradical. We recall a pictorial way to represent elements of $\mathfrak{n}$ and in particular, to obtain an element of the Richardson orbit $\mathcal{O}(d)$. This can be found in [6, Section 2] and in [1, Section 3]. We draw $t$ top-adjusted columns of $d_{1}, d_{2}, \ldots, d_{t}$ vertices. The vertices are connected using edges between vertices of different columns. If two vertices lie on the same height and there is no third vertex between them on that height then we call the two vertices neighbors. The complete line diagram for $d, L_{R}(d)$, is the diagram with horizontal edges between all neighbored vertices (as the second and the third diagram of Example 2.1). A line diagram $L(d)$ for $d$ is a diagram with arbitrary edges between different columns (possibly with branching). A collection of connected edges is called a chain of edges (see the example below). If no branching occurs in a line diagram then a chain consisting of $l$ edges connects $l+1$ vertices. In that case we can define the length of a chain: the length of a chain of edges in a line diagram (without branching) is the number of edges the chain contains. A chain of length 0 is a vertex that is not connected to any other vertex.

In Example 2.1, we show two complete and a branched line diagram for $d=(3,1,2,4)$ resp. for $d=(3,1,6,1,2,5,4)$.

Example 2.1 (a) A line diagram with branching and the complete line diagram $L_{R}(d)$ for $d=(3,1,2,4)$ are here. To the right of the latter we give the lengths of the chains in the diagram.
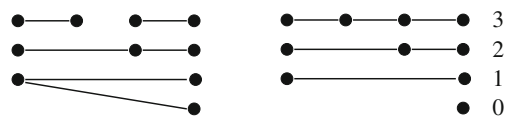
(b) Now we consider our running example $d=(7,5,2,3,5,1,2,6,5)$. Its complete line diagram $L_{R}(d)$ is here, with the lengths of the chains to the right.

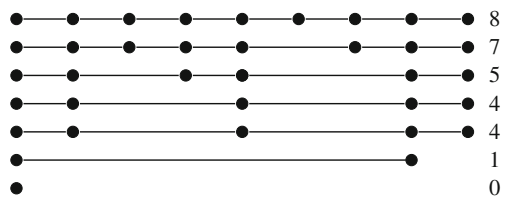

We will see in the next subsection that the line diagram $L_{R}(d)$ determines an element of the Richardson orbit of $\mathfrak{n}$. In general, line diagrams give rise to elements of the nilradical of nilpotency class smaller than $\lambda=\lambda(d)$ with respect to the Bruhat order.

Any line diagram (complete or not) gives rise to an element $A$ of $\mathfrak{n}$ :

The sizes of the columns of a line diagram correspond to the sizes of the square blocks in the Levi factor of $\mathfrak{p}$. An edge between column $i$ and column $j$ (with $i<j$ ) of the diagram corresponds to a non-zero entry in the block $A_{i j}$ of the matrix $A$. A chain of two joint edges between three columns $i_{0}<i_{1}<i_{2}$ gives rise to a non-zero entry in block $A_{\left(i_{0}, i_{2}\right)}^{2}$ of the matrix $A^{2}$, etc. This can be made explicit, as we explain in the next subsection.

\subsection{From line diagrams to the nilradical}

The elements of the nilradical $\mathfrak{n}$ for the dimension vector $d=\left(d_{1}, \ldots, d_{t}\right)$ are nilpotent endomorphisms of $k^{n}$, for $n=\sum d_{i}$. In particular, if we write $e_{1}, \ldots, e_{n}$ for a basis of $k^{n}$, then the elements of $\mathfrak{n}$ are sums $\sum_{i<j} a_{i j} E_{i j}$ for some $a_{i j} \in k$ where the elementary matrix $E_{i j}$ sends $e_{j}$ to $e_{i}$

We now describe a map associating an element of the nilradical to a given line diagram. We view the vertices of a line diagram $L(d)$ as labelled by the numbers $1,2, \ldots, n$, starting at the top left vertex, with $1,2, \ldots, d_{1}$ in the first column, $d_{1}+1, \ldots, d_{1}+d_{2}$ in the second column, etc. Now if two vertices $i$ and $j$ (with $i<j$ ) are joint by an edge, we associate to this edge the matrix $E_{i j}$.

We denote an edge between two vertices $i$ and $j(i<j \leq n)$ of the diagram by $e(i, j)$. Then we associate to an edge $e(i, j)$ of $L(d)$ the elementary matrix $E_{i j} \in \mathfrak{n}$. This can be extended to a map from the set of line diagrams for $d$ to the nilradical $\mathfrak{n}$ by linearity.

For later use, we denote this map by $\Phi$ :

$$
\Phi:\{\text { line diagrams for } d\} \longrightarrow \mathfrak{n}, L(d) \mapsto \sum_{e(i, j) \in L(d)} E_{i j}
$$

If $L(d)$ is a line diagram without branching, then the partition of the image under $\Phi$ of the line diagram $L(d)$ can be read off from it directly as follows: if $L(d)$ has $s$ chains of lengths $c_{1}, c_{2}, \ldots, c_{s}($ all $\geq 0)$. Then $\sum_{j=1}^{s}\left(c_{j}+1\right)=\sum_{i=1}^{t} d_{i}=n$.

Remark 2.2 Let $L(d)$ be a line diagram without branching and let $c_{1}, \ldots, c_{s}$ be the lengths of the chains of $L(d)$. Let $\mu=\left(\mu_{1}, \ldots, \mu_{s}\right)$ be the partition obtained by ordering the numbers $c_{j}+1$ by size. Then $\mu$ is the partition of $\Phi(L(d))$.

In particular, $\Phi\left(L_{R}(d)\right)$ is an element of the Richardson orbit $\mathcal{O}(d)$ since the partition of $L_{R}(d)$ is just the dual of the dimension vector $d$ and this is equal to $\lambda(d)$ (cf. Section 3 in [1]). If $L(d)$ is any other line diagram for $d L(d)$ (without branching), with lengths of chains $c_{1}, \ldots, c_{s}$ and $\mu_{i}:=c_{i}+1$ then we always have $\sum_{j=1}^{k} \mu_{j} \leq \sum_{j=1}^{k} \lambda_{j}(d)$ and so the 
partition of $\Phi(L(d))$ is smaller than or equal to the partition of $\Phi\left(L_{R}(d)\right)$ under the Bruhat order.

To summarize, we have the following:

Lemma 2.3 Let d be a dimension vector. Then, $\Phi(L(d))$ is an element of the nilradical $\mathfrak{n}$ of nilpotency class $\mu \leq \lambda(d)$. In other words, $\Phi(L(d))$ lies in $\mathfrak{n} \cap C(\mu)$.

Example 2.4 Let $d=(3,1,2,4)$ as in Example 2.1(a). The lengths of the chains of $L_{R}(d)$ are $3,2,1,0$, the Richardson orbit has partition $(4,3,2,1)$. We compute the matrix of the complete line diagram $L_{R}(d)$, and the powers of this matrix. Let $X(d):=\Phi\left(L_{R}(d)\right)$. Then $X(d)$ and its powers are

$$
\begin{aligned}
& X(d)=E_{14}+E_{45}+E_{57}+E_{26}+E_{68}+E_{39} \\
& X(d)^{2}=E_{15}+E_{47}+E_{28} \\
& X(d)^{3}=E_{17} \\
& X(d)^{k}=0 \text { for } k>3 .
\end{aligned}
$$

Recall that we have defined the varieties $Z_{i j}^{k}$ by comparing the ranks of certain submatrices of elements in the nilradical $\mathfrak{n}$ to the corresponding rank $r_{i j}^{k}$ of a Richardson element, $\mathrm{cf}$. Definition 1.1. We thus need to be able to compute the rank of the submatrix $X(d)[i j]$ of an element $X(d)$ of the Richardson orbit $\mathcal{O}(d)$ and of its powers. For this, we can use the line diagram $L_{R}(d)$. Let $X(d)=\sum_{e(k, l) \in L_{R}(d)} E_{k l}$ be the Richardson element given by $L_{R}(d)$.

To compute the rank $r_{1 t}^{k}$ of $X(d)^{k}$, it is enough to count the chains of length $\geq k$ in the line diagram $L_{R}(d)$. Analogously, to find the rank $r_{i j}^{k}$ of the $k$ th power of the submatrix $X(d)[i j]$, one has to count the chains of length $\geq k$ between the $i$ th and $j$ th column in $L_{R}(d)$ :

Let $1 \leq k<l \leq n$ be such that the image $\Phi(e(k, l))$ of the edge $e(k, l)$ is in $X(d)[i j]$. That means we are considering edges $e(k, l)$ starting in some column $i_{1} \geq i$ and ending in some column $i_{2} \leq j$. Thus, in computing $r_{i j}^{k}$, we really consider the $k$ th power of the matrix which arises from columns $i, i+1, \ldots, j$ of $L_{R}(d)$. We now introduce the notation to refer to the subdiagram consisting of these columns. We denote by $L_{R}(d)[i j]$ subdiagram of $L_{R}(d)$ of all vertices from the $i$ th up to the $j$ th column and of all edges starting strictly after the $(i-1)$ st column resp. ending strictly before the $(j+1)$ st column. In other words, we remove columns $1,2, \ldots, i-1$ and columns $j+1, \ldots, t$ together with all edges incident with them.

With this notation we have

$$
r_{i j}^{k}=\#\left\{\text { chains in } L_{R}(d)[i j] \text { with at least } k \text { edges }\right\}
$$

for $1 \leq i<j \leq t, k \geq 1$.

Similarly, if $L(d)$ is a line diagram for $d$, we write $L(d)[i j]$ to denote the subdiagram of $L(d)$ of rows $i$ to $j$.

Example 2.5 The subdiagram $L_{R}(d)$ [47] for $d=(7,5,2,3,5,1,2,6,5)$ of the diagram $L_{R}(d)$ from (b) of Example 2.1 is shown here (dotted lines and empty circles are thought to be removed):

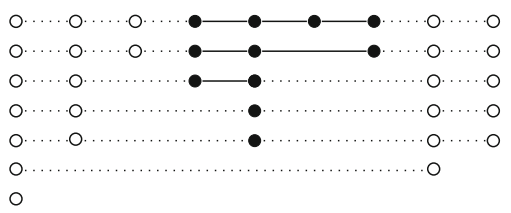


Fig. 2 Our running example has $d_{<}[26]=\emptyset$, so $\kappa(2,6)=4$

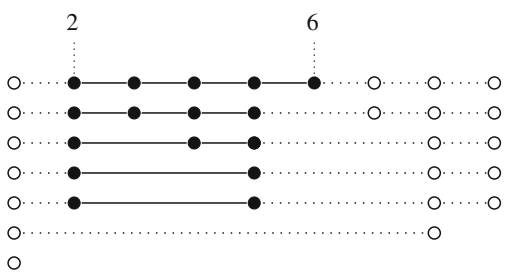

\subsection{The varieties $Z_{i j}$}

As explained earlier, we want to show that the irreducible components of $Z$ are indexed by the parameter set $\Lambda(d)$. With this in mind, we now discuss the properties of the varieties $Z_{i j}^{k}$. We will prove that for $l \neq \kappa(i, j), Z_{i j}^{l}$ is either empty or contained in $Z_{i j}$ or in the union $Z_{i j_{0}} \cup Z_{i_{0} j}$ for some $i_{0} \leq j_{0}$. Later in this section we will see that not all $(i, j)$ with $1 \leq i<j \leq t$ are needed to describe the complement $Z$.

The following notations will be useful:

$$
\begin{aligned}
& d_{<}[i j]:=\left|\left\{l \mid i<l<j, d_{l}<\min \left(d_{i}, d_{j}\right)\right\}\right| \\
& d_{\geq}[i j]:=\left|\left\{l \mid i<l<j, d_{l} \geq \min \left(d_{i}, d_{j}\right)\right\}\right| .
\end{aligned}
$$

If $d=(7,5,2,3,5,1,2,6,5)$, then $d_{<}[25]=2, d_{<}[26]=\emptyset$ and $d_{\geq}[26]=3$.

Remark 2.6 Observe that

$$
\begin{aligned}
\kappa(i, j) & =1+\# d_{\geq}[i j] \\
& =j-i-\# d_{<}[i j] .
\end{aligned}
$$

In particular, $\kappa(i, j)=j-i$ if and only if $d_{<}[i j]=\emptyset$. Figure 2 illustrates this.

Lemma 2.7 Let $d=\left(d_{1}, \ldots, d_{t}\right)$ be a dimension vector and $1 \leq i<j \leq t$. Then for $k>0$ we have

$$
Z_{i j}^{k}=\emptyset \text { if and only if } k>j-i .
$$

Proof One has $r_{i j}^{k}=\operatorname{rk} X(d)[i j]^{k}>0$ exactly for $k \leq j-i$ and $0 \in Z_{i j}^{k}$ if and only if $r_{i j}^{k}>0$.

It remains to consider the cases where $l$ is smaller than $\kappa(i, j)$ or when $l$ lies between $\kappa(i, j)$ and $j-i$. This is covered by the next two statements.

Lemma 2.8 For $1 \leq l<\kappa(i, j)$ the following holds:

$$
Z_{i j}^{l} \subsetneq Z_{i j}
$$

Proof We may assume $d_{i} \leq d_{j}$. For any $B \in \mathfrak{n}$ the rank of $B[i j]^{l}$ is independent of the order of $d_{i}, d_{i+1}, \ldots, d_{j}$ : in computing the rank, we need to know the number of (independent) chains of length $l$ in the line diagram of $b[i j]$. Hence we may reorder $d_{i}, \ldots, d_{j}$ to obtain $d_{s_{1}}, \ldots, d_{s_{j-i+1}}$ with $d_{s_{k}} \leq d_{s_{k+1}}$ for $k=1, \ldots, j-i$. One computes $r_{i j}^{l}=\operatorname{rk} X(d)[i j]^{l}$ as the sum $\sum_{k=0}^{j-i-l} d_{i+k}$.

Let $A$ belong to $Z_{i j}^{l}$ for some $l<\kappa(i, j)$. Thus rk $A[i j]^{l}<r_{i j}^{l}=\operatorname{rk} X(d)[i j]^{l}$. But then also the rank of $A[i j]^{k}$ is smaller than $r_{i j}^{k}$ for $k=l+1, \ldots, \kappa(i, j)$. In particular, $A \in Z_{i j}$. The inequality is clear. 
Fig. 3 The case $\left|d_{<}[i j]\right|=1$ : in the running example, we have $d_{<}[47]=\{6\}$ and $\kappa(i, j)=2$
Fig. 4 The case $i_{0} \neq j_{0} \in d_{<}[i j]$ : our running example has $d_{<}[48]=\{6,7\}$ and so $\kappa(4,8)=2$
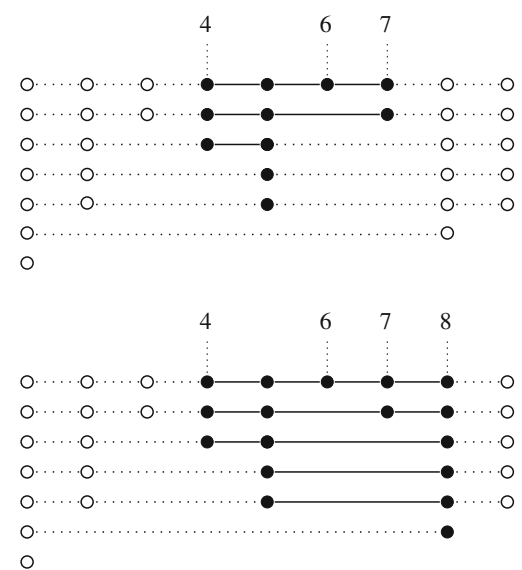

Let $A$ belong to $Z_{i j}^{l}$ for some $l<\kappa(i, j)$. Thus rk $A[i j]^{l}<r_{i j}^{l}$. But then also the rank of $A[i j]^{k}$ is smaller than $r_{i j}^{k}$ for $k=l+1, \ldots, \kappa(i, j)$. In particular, $A \in Z_{i j}$.

Lemma 2.9 For $\kappa(i, j)<l \leq j-i$ the following holds: there exist $i_{0} \leq j_{0} \in$ $d_{<}[i j], d_{i_{0}}, d_{j_{0}}<\min \left(d_{i}, d_{j}\right)$ maximal, such that

$$
Z_{i j}^{l} \subseteq Z_{i j_{0}} \cup Z_{i_{0} j}
$$

Proof We first observe that for elements of the Richardson orbit, the rank $r_{i j}^{l}$ is

$$
r_{i j}^{l}=\sum_{i_{0}=i}^{j-l} \max _{i_{0}<\cdots<i_{l} \leq j} \min \left\{d_{i_{0}}, \ldots, d_{i_{l}}\right\}
$$

(1) Let us first consider the case where $d_{<}[i j]$ only has one element, say $d_{<}[i j]=\left\{i_{0}\right\}$, see Fig. 3. Then $\kappa(i, j)=j-i-1$ and so $l=j-i$.

For $A \in \mathfrak{n}$ to be an element of $Z_{i j}^{l}$, the rank of $A[i j]^{l}$ is smaller than $r_{i j}^{l}$. Since $d_{i_{0}}$ is minimal among all $d_{i}, \ldots, d_{j}$, this implies rk $A\left[i i_{0}\right]^{l}<r_{i j}^{l}$ or $\mathrm{rk} A\left[i_{0} j\right]^{l}<r_{i j}^{l}$ and we are done.

(2) The case where $d_{<}[i j]$ has at least two elements only needs a slight modification of the argument. Take $i_{0}, j_{0}$ from $d_{<}[i j]$ with $d_{i_{0}}, d_{j_{0}}$ maximal with $i_{0}$ being the smallest among these indices, $j_{0}$ the largest one (we do not distinguish between the two possibilities $d_{i_{0}}=d_{j_{0}}$ and $d_{i_{0}} \neq d_{j_{0}}$ ), see Fig. 4 . With a similar reasoning as in part (1) of the proof, $A$ then lies in $Z_{i, j_{0}}$ or in $Z_{i_{0}, j}$.

Lemma 2.10 The complement $Z$ decomposes as follows:

$$
Z=\cup_{1 \leq i<j \leq t} Z_{i j}=\cup_{i j} \cup_{k \geq 1} Z_{i j}^{k} .
$$

Proof The inclusion $\subseteq$ of the second equality is clear. To obtain the inclusion $\supseteq$, one uses Lemmata 2.7-2.9. Consider the first equality: by definition, $A \in Z$ if and only if $A \notin \mathcal{O}(d)$. The latter is the case if and only if there exist $1 \leq i<j \leq t, k \leq j-i$, such that $A \in Z_{i j}^{k}$ : to see this, one uses the formula for the dimension of the stabilizer of $A \in \mathfrak{g l}_{n}$, see [11]. This formula uses the dimensions of the kernels of the maps $A^{k}, k \geq 1$. The stabilizer of $A$ has dimension 0 if and only if $A$ is an element of $\mathcal{O}(d)$. 

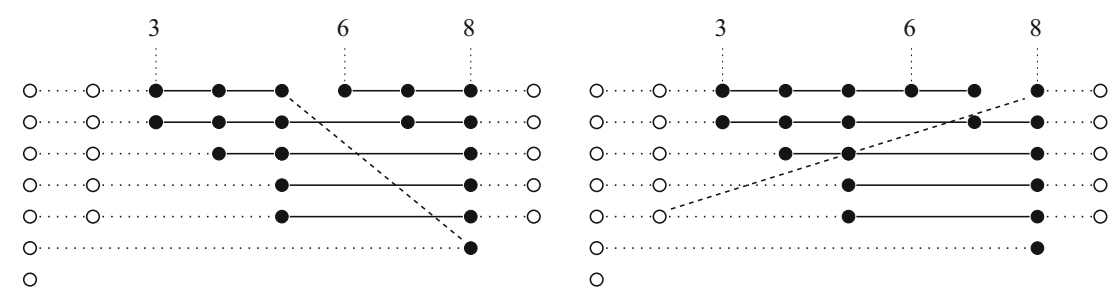

Fig. 5 Examples for $A \in Z_{i l}$ resp. for $A \in Z_{l j}$ for $d=(7,5,2,3,5,1,2,6,5)$, with $i=3, j=8$ and $l=6$

It now remains to see that the $(i, j) \in \Lambda(d)$ are enough to describe the irreducible components of $Z$. In a first step (Lemma 2.11), we start with $(i, j) \notin \Gamma(d)$ and show that in that case $Z_{i j}$ is contained in a union of $Z_{k l}$ 's such that the corresponding $(k, l)$ all lie in $\Gamma(d)$.

Then we consider an element $(i, j)$ of $\Gamma(d) \backslash \Lambda(d)$ and show that we can find $(k, l) \in \Lambda(d)$ with $Z_{i j} \subseteq Z_{k l}$ (Lemma 2.12 and Corollary 2.13). As always, we assume that $1 \leq i<j \leq t$ and $1 \leq k<l \leq t$.

Lemma 2.11 Assume that $(i, j)$ does not belong to $\Gamma(d)$. Then there exists $\Gamma^{\prime}(d) \subseteq \Gamma(d)$ such that

$$
Z_{i j} \subseteq \bigcup_{(k, l) \in \Gamma^{\prime}(d)} Z_{k l}
$$

Proof It is enough to show that we can find an $l, i<l<j$, with $\min \left(d_{i}, d_{j}\right) \leq d_{l} \leq$ $\max \left(d_{i}, d_{j}\right)$, such that

$$
Z_{i j} \subseteq Z_{i l} \cup Z_{l j}
$$

By iterating this, we will eventually end up with a subset $\Gamma^{\prime}(d) \subset \Gamma(d)$ as in the statement of the lemma.

So choose an $l, 1<l<t$, with $\min \left(d_{i}, d_{j}\right) \leq d_{l} \leq \max \left(d_{i}, d_{j}\right)$ (such an $l$ exists since $(i, j) \notin \Gamma(d))$. Take $A \in Z_{i j}$ arbitrary. By assumption, $A[i j]^{\kappa(i, j)}$ is defective, i.e. rk $A[i j]^{\kappa(i, j)}<r_{i j}^{\kappa(i, j)}$. Since $d_{l} \geq d_{i}, d_{j}$, the defectiveness is inherited from $A[i l]$ or from $A[l j]$ and $A \in Z_{i l}$ or $A \in Z_{l j}$ accordingly.

Let us remark that when removing an edge of a chain of $L_{R}(d)$ in the proof above, we ensured that the matrix $A$ has a zero entry at the corresponding position. In general, the diagram of a matrix in $Z_{i l}$ resp. in $Z_{l j}$ has more non-zero entries than the ones obtained after removing one edge from $L_{R}(d)$ : this is illustrated by the dashed lines in Fig. 5.

The following lemma states that for any $(i, j)$ from $\Gamma(d) \backslash \Lambda(d)$ there exists $(k, l)$ from $\Lambda(d)$ with $k \leq i<j \leq l$ such that $Z_{i j} \subseteq Z_{k l}$.

Lemma 2.12 Assume that $(i, j) \in \Gamma(d) \backslash \Lambda(d)$. Then one of the following holds:

there exists $k>j$ with $Z_{i j} \subseteq Z_{i k}$

or there exists $l<i$ with $Z_{i j} \subseteq Z_{l j}$.

Proof First observe that $d_{i} \neq d_{j}$ since $(i, j)$ belongs to $\Lambda(d)$ otherwise. Without loss of generality, we assume $d_{i}<d_{j}$. We have three cases to consider:

(i) There is $k_{1} \in\{1, \ldots, i-1\} \cup\{j+1, \ldots, t\}$ with $d_{i}<d_{k_{1}}<d_{j}$.

(ii) There exists $k_{2}<i$ with $d_{k_{2}}=d_{j}$.

(iii) There exists $k_{3}>j$ with $d_{k_{2}}=d_{i}$. 
Fig. 6 For the running example, $(7,8)$ is in $\Gamma(d) \backslash \Lambda(d)$, as for all $i, d_{m_{i}}$ violates the assumptions on $\Lambda(d)$

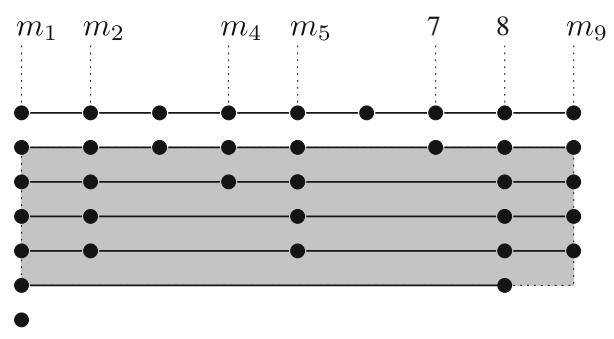

The three cases are illustrated in Fig. 6: if $(i, j) \in \Gamma(d)$ but not in $\Lambda(d)$ then one of the following has to occur: there has to be a $k$ with $d_{k}$ inside the shaded area or with $d_{k}$ lying on the same height as $d_{j}$ (if $k<i$ ) resp. on the same height as $d_{i}$ (if $k>j$ ).

Case (i) with $k_{1}>j$ : among the $k_{1}>j$ with $d_{i}<d_{k_{1}}<d_{j}$ choose one with $d_{k_{1}}-d_{i}$ minimal, and $k_{1}$ minimal (i.e. as close to $j$ as possible). Note that we have $\kappa(i, j) \leq \kappa\left(i, k_{1}\right)$. Now $A \in Z_{i j}$ means that $A[i j]^{\kappa(i, j)}$ is defective. Since $d_{i}<d_{k_{1}}$, this defectiveness has to be inherited from $A\left[i, k_{1}\right]$, i.e. rk $A\left[i, k_{1}\right]^{\kappa\left(i, k_{1}\right)}<r_{i, k_{1}}^{\kappa\left(i, k_{1}\right)}$ and so, $Z_{i j} \subseteq Z_{i, k_{1}}$.

Case (i) with $k_{1}<i$ : here, we choose $k_{1}$ accordingly to be such that $d_{j}-d_{k_{1}}$ is minimal and $k_{1}<i$ maximal among those (i.e. as close to $i$ as possible). One checks that $\kappa(i, j) \leq \kappa\left(k_{1}, j\right)$. Similarly as before, one gets $Z_{i j} \subseteq Z_{k_{1}, j}$.

Case (ii): among the $k_{2}<i$ with $d_{k_{2}}=d_{j}$, choose the maximal one (i.e. the one closest to $i$ ). We have $\kappa(i, j) \leq \kappa\left(k_{2}, j\right)$ and we get $Z_{i j} \subseteq Z_{k_{2}, j}$. Case (iii) is completely analogous to case (ii).

Observe that $\left(k_{2}, j\right)$ and $\left(i, k_{3}\right)$ from cases (ii) and (iii) above are elements of $\Lambda(d)$.

Corollary 2.13 For any $(i, j) \in \Gamma(d) \backslash \Lambda(d)$ there exists $(k, l) \in \Lambda(d)$ such that

$$
Z_{i j} \subseteq Z_{k l}
$$

Proof Without loss of generality, we can assume $d_{i}<d_{j}$. By the observation after the proof of Lemma 2.12, we are done if there exists $k^{\prime}<i$ with $d_{k^{\prime}}=d_{j}$ or $k^{\prime \prime}>j$ with $d_{k^{\prime \prime}}=d_{i}$. Using similar arguments, one sees that if there exist $k^{\prime}<i$ and $k^{\prime \prime}>j$ with $d_{i}<d_{k^{\prime}}=d_{k^{\prime \prime}}<d_{j}$ then $\left(k^{\prime}, k^{\prime \prime}\right) \in \Lambda(d)$ and $Z_{i j} \subseteq Z_{k^{\prime}, k^{\prime \prime}}$. Thus, assume that there exists $k \in\{1, \ldots, i-1\} \cup\{j+1, \ldots, t\}$ with $d_{i}<d_{k}<d_{j}$ and such that there is no $k^{\prime}<i$ with $d_{k^{\prime}}=d_{j}$ and no $k^{\prime \prime}>j$ with $d_{k^{\prime \prime}}=d_{i}$.

If $k>j$, we choose $k$ such that $d_{k}-d_{i}$ is minimal and take the minimal $k>j$ among these (i.e. $k$ is as close to $j$ as possible). There are two possibilities: either we have $d_{k^{\prime}}>d_{k}$ for all $k^{\prime}<i$. Then, $\left(k^{\prime}, k\right) \in \Lambda(d)$ and one checks that $Z_{i j} \subseteq Z_{k^{\prime}, k}$.

Or there exists is $k^{\prime}<i$ with $d_{i}<d_{k^{\prime}}<d_{k}$. In that case, among the $k^{\prime}<i$ with this property, we choose one with $d_{k}-d_{k^{\prime}}$ minimal and such that $k^{\prime}<i$ is maximal (i.e. $k^{\prime}$ is as close to $i$ as possible). Again, we get $\left(k^{\prime}, k\right) \in \Lambda(d)$ and $Z_{i j} \subseteq Z_{k^{\prime}, k}$.

The case $k<i$ is analogous.

\section{Components via tableaux}

Let $d=\left(d_{1}, \ldots, d_{t}\right)$ be a composition of $n$ and $\mathcal{O}(d)$ be the corresponding Richardson orbit in $\mathfrak{n}$, let $\lambda=\lambda(d)$ be the partition of the Richardson orbit. The second description of the irreducible components of $Z=\mathfrak{n} \backslash \mathcal{O}(d)$ uses partitions $\mu_{i j}$, for $(i, j) \in \Lambda(d)$ and tableaux corresponding to them. Observe that $\lambda_{1}=t$, that $\lambda_{2}$ is the number of $d_{i} \geq 2$ appearing in $d, \lambda_{3}=\#\left\{d_{i} \mid d_{i} \geq 3\right\}$, and so on. 
Let us introduce the necessary notation. If $\lambda=\lambda_{1} \geq \lambda_{2} \geq \cdots \geq \lambda_{s} \geq 1$ is a partition of $n$ we will also use $\lambda$ to denote the Young diagram of shape $\lambda$. It has $s$ rows, with $\lambda_{1}$ boxes in the top row, $\lambda_{2}$ boxes in the second row, etc., up to $\lambda_{s}$ boxes in the last row. That means that we view Young diagrams as a number of right adjusted rows of boxes, attached to the top left corner, and decreasing in length from top to bottom. A standard reference for this is the book [8] by Fulton.

\subsection{The Young tableaux $\mathcal{T}(\mu, d)$}

Let $\mu \leq \lambda(d)$ be a partition of $n$ (unless mentioned otherwise, we will always deal with partitions of $n$ ).

Definition 3.1 We define a Young tableau of shape $\mu$ and of dimension vector $d$ to be a filling of the Young diagram of $\mu$ with $d_{1}$ ones, $d_{2}$ twos, etc. We write $\mathcal{T}(\mu, d)$ for the set of all Young tableaux of shape $\mu$ and for $d$.

Recall that the rules for fillings of a Young diagram are that the numbers in a row strictly increase from left to right and that the numbers in a column weakly increase from top to bottom. In general, there might be several Young tableaux of a given shape for a given $d$. There is exactly one Young tableau of shape $\lambda=\lambda(d)$ and for $d$, so $\mathcal{T}(\lambda(d), d)$ only has one element. To abbreviate, we will just call it $T(d)$. The entries of the boxes of its first row are $1,2, \ldots, t$.

Example 3.2 The partition of the composition $d=(7,5,2,3,5,1,2,6,5)$ of 36 is $\lambda(d)=$ $(9,8,6,5,5,2,1)$. The partition $\mu=(9,8,6,5,4,3,1)$ is smaller than $\lambda(d)$ and $\mathcal{T}(\mu, d)$ consists of one element $T(\mu, d)$. We include $T(d)$ and $T(\mu, d)$ here.
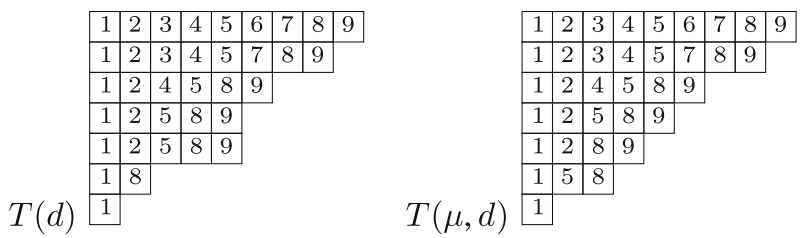

In order to understand the irreducible components of the complement $Z=\mathfrak{n} \backslash \mathcal{O}(d)$, we have to consider the intersections $\mathfrak{n} \cap C(\mu)$ for $\mu<\lambda(d)$. Each irreducible component of $Z$ corresponds to an irreducible component in such an intersection. Here, we can use a result of the second author (cf. Section 4.2 of [10]). First, one observes that the irreducible components of $\mathfrak{n} \cap C(\mu)$ are given by sequences $\mu^{1}, \ldots, \mu^{t}$ where $\mu^{i}$ is a partition of $\sum_{j}^{i} d_{j}$ where $\mu^{t}=\mu$ and such that $0 \leq \mu_{j}^{i+1}-\mu_{j}^{i} \leq 1$ (for all $j$, for $1 \leq i<t$ ). And the latter correspond to tableaux of shape $\mu$ with $d_{i}$ entries $i$, i.e. the elements of $\mathcal{T}(\mu, d)$ in our notation.

Proposition 3.3 Let $\mu \leq \lambda(d)$ be a partition of $n$. Then the irreducible components of $\mathfrak{n} \cap C(\mu)$ are in natural bijection with with the tableaux in $\mathcal{T}(\mu, d)$.

Proof This is Satz 4.2.8 in [10].

Example 3.4 Let $d=\left(d_{1}, \ldots, d_{t}\right)$ be a dimension vector and $\lambda=\lambda(d)$. We know that $\mathfrak{n} \cap C(\lambda)=\mathcal{O}(d)$ is the Richardson orbit. On the other hand, $\mathcal{T}(\lambda, d)=T(d)$ has exactly one tableau. We now explain how to relate the complete line diagram $L_{R}(d)$ to the tableau $T(d)$. The lengths of the chains in $L_{R}(d)$ are the entries of the partition of $\lambda$ and hence give 
the shape of $T(d)$. The filling of $T(d)$ can now be obtained from $L_{R}(d)$ by labelling each vertex of the $i$ th column in $L_{R}(d)$ by an $i$. These numbers are then copied row by row, from left to right into the Young diagram of shape $\lambda$ to get $T(d)$.
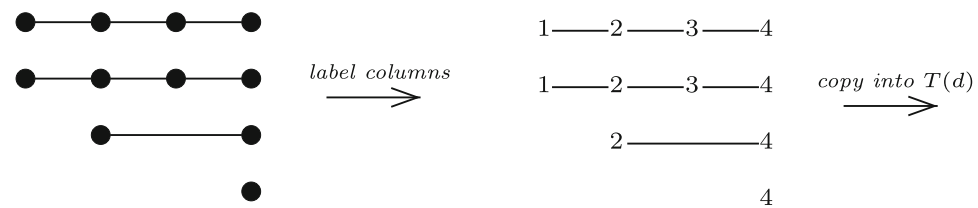

\begin{tabular}{|l|l|l|l|}
\hline 1 & 2 & 3 & 4 \\
\hline 1 & 2 & 3 & 4 \\
\cline { 1 - 2 } 2 & 4 & \multicolumn{2}{|c}{} \\
\cline { 1 - 2 } 4 & \multicolumn{2}{|c}{} \\
\cline { 1 - 1 } &
\end{tabular}

From this connection between the line diagram $L_{R}(d)$ and $T(d)$ one deduces the following useful observation. Every pair $(i, j)$ with $1 \leq i<j \leq t$ determines a unique row of $T(d)$ namely the last row of $T(d)$ containing $i$ and $j$. Such a row always exists as the first row just consists of the boxes with numbers $1,2,3, \ldots, t$. We denote this row by $s(i, j)$.

Lemma 3.5 The number of boxes between $i$ and $j$ in $\operatorname{row} s(i, j)$ of $T(d)$ is equal to $\kappa(i, j)-1$.

Proposition 3.3 describes the irreducible components of the intersections $\mathfrak{n} \cap C(\mu)$ for $\mu \leq \lambda$ : they are given by the Young tableaux in $\mathcal{T}(\mu, d)$, i.e. by all possible fillings of the diagram $\mu$ by the numbers given by $d$.

Clearly, not all irreducible components of the different intersections $\mathfrak{n} \cap C(\mu)$ give rise to an irreducible component of $Z$. If $\mu_{2} \leq \mu_{1}$ and $T_{i} \in \mathcal{T}\left(\mu_{i}, d\right)$ are tableaux such that $T_{2}$ can be obtained from $T_{1}$ by moving down boxes successively, then the irreducible component corresponding to $T_{2}$ is already contained in the irreducible component corresponding to $T_{1}$ and thus does not give rise to a new irreducible component of the complement $Z$ of the Richardson orbit. This is in particular the case, if $T_{1}$ is obtained from the tableau $T(d)$ of the Richardson orbit by moving down a single box and $T_{2}$ is a degeneration of $T_{1}$ (obtained by moving down boxes from $T_{1}$ ). Thus, the only candidates for irreducible components are the ones given by tableaux which can be obtained from $T(d)$ by moving down a single box to the closest possible row. We call such a degeneration a minimal movement.

\subsection{The Young tableaux $T(i, j)$}

To describe minimal movements, we now define certain tableaux $T(i, j)$.

Definition 3.6 The tableau $T(i, j)$ is the tableau obtained from $T(d)$ by removing the box containing the number $j$ from row $s(i, j)$ and inserting it in the nearest row in order to obtain another tableau. In other words: among the possible rows where this box could be inserted, we choose the one that is closest to row $s(i, j)$. We denote the partition of the resulting tableau $T(i, j)$ by $\mu(i, j)$.

Definition 3.7 For a tableau $T(i, j)$ we define $\mathfrak{n}(T(i, j)) \subseteq \mathfrak{n}$ to be the irreducible component of $\mathfrak{n} \cap C(\mu(i, j))$ whose tableau is $T(i, j)$.

We claim that $\mathfrak{n}(T(i, j))$ gives rise to an irreducible component of the complement $Z$ exactly when $(i, j)$ belongs to the parameter set $\Lambda(d)$.

For completeness, we recall the definition of a the tableau $T$ for a an irreducible component in $C(\mu) \cap \mathfrak{n}$. Consider a maximal flag $V_{0} \subset V_{1} \subset \cdots \subset V_{t}$ of vector spaces that is stabilized by $P(d)$. Take any matrix $A$ in the open subset of an irreducible component of $C(\mu) \cap \mathfrak{n}$ where $A$ restricted to $V_{i}$ has constant Jordan type. Then the Young diagram 


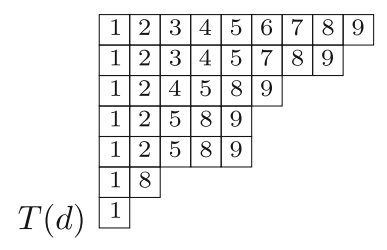
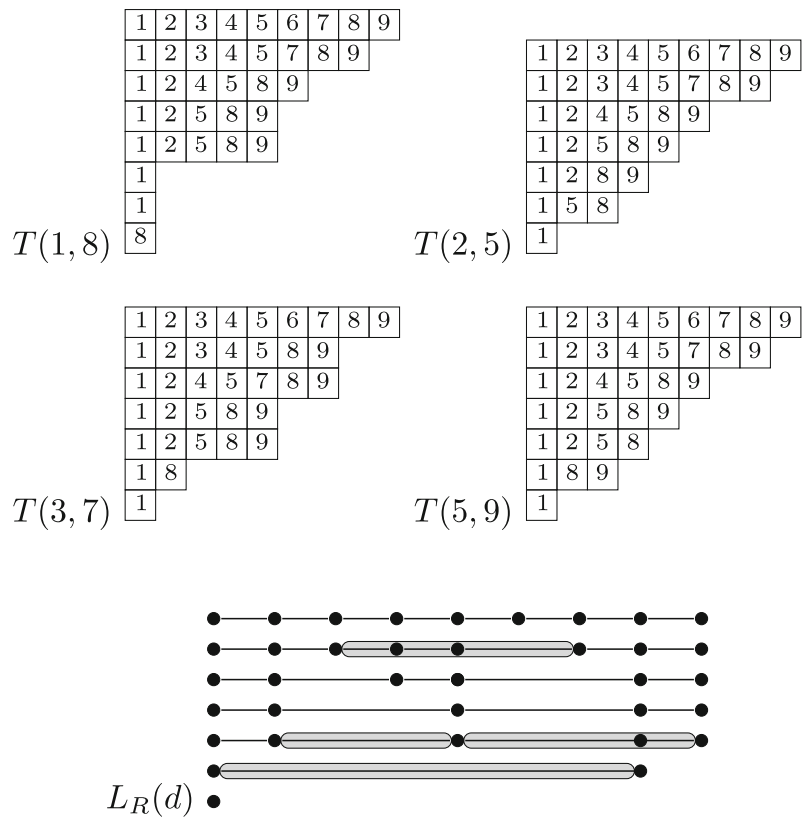

Fig. 7 The tableaux $T(d), T(i, j)$ and $L_{R}(d)$ for Example 3.8

of $\left.A\right|_{V_{i}}$ is the partition obtained from $T$ by deleting all boxes with entries $i+1, \ldots, t$. So the subdiagram consisting of all boxes with entries at most $i$ measures the generic Jordan type of $A$ restricted to the subspace $V_{i}$. In particular, the equation defining the component corresponding to $T(i, j)$ can involve only equations in the entries of $A[1, j]$. Even stronger, we will see in Lemma 3.9 that the equations involve only entries in $A[i, j]$ for $(i, j)$ in $\Gamma(d)$.

To prepare for Lemma 3.9 we observe that for $(1, t) \in \Gamma(d)$ the component $\mathfrak{n}(T(1, t))$ coincides with $C(\mu(1, t)) \cap \mathfrak{n}$ since there is only one tableau for the partition $\mu(1, t)$ with dimension vector $d$. Consequently, this component is defined by the equation rk $A[1, t]^{\kappa(1, t)}<r_{1, t}^{\kappa(1, t)}$ defining $C(\mu(1, t)) \cap \mathfrak{n}$ inside $\mathfrak{n}$.

By definition, the tableau $T(i, j)$ is obtained from $T(d)$ through a minimal movement. Its partition $\mu(i, j)$ is clearly smaller than $\lambda=\lambda(d)$ as the lengths of the rows of a tableau are the parts of the corresponding partition. In particular, these lengths form a decreasing sequence of positive numbers. Thus, moving down a box from a row of length $k$ to a lower row of length at most $k-2$ results in a partition which is smaller than the original partition. Note, however, that different elements $(i, j)$ and $(k, l)$ can lead to the same partition $\mu(i, j)=\mu(k, l)$, e.g. $\mu(2,5)=\mu(5,9)$ in Example 3.8 below.

Example 3.8 Let $d=(7,5,2,3,5,1,2,6,5)$ be a dimension vector, $n=36$. To illustrate the construction of $T(i, j)$ we compute these tableaux for all $(i, j) \in \Lambda(d)=$ $\{(1,8),(2,5),(3,7),(5,9)\}$. They are presented in Fig. 7 . In the picture showing the line 
diagram $L_{R}(d)$ we have indicated the connections between the columns $i$ and $j$ for all $(i, j) \in \Lambda(d)$ by shaded areas.

Lemma 3.9 Let $d=\left(d_{1}, \ldots, d_{t}\right)$ be a dimension vector, $(i, j) \in \Gamma(d)$. Then

$$
\mathfrak{n}(T(i, j))=Z_{i j} .
$$

In particular, $Z_{i, j}$ is irreducible.

Proof We show that $\mathfrak{n}(T(i, j))=\left\{A \in \mathfrak{n} \mid \operatorname{rk} A[i j]^{\kappa(i, j)}<r_{i j}^{\kappa(i, j)}\right\}=Z_{i j}$. The second equation holds as it is the definition of $Z_{i j}$.

We first prove the lemma for a special case: replace $d_{1}, \ldots, d_{i-1}$ and $d_{j+1}, \ldots, d_{t}$ by zero, thus we get a new shorter dimension vector $e:=\left(d_{i}, \ldots, d_{j}\right)=\left(e_{1}, \ldots, e_{j-i+1}\right)$. Note that $(i, j)$ is in $\Gamma(d)$ precisely when $(1, j-i+1)$ is in $\Gamma(e)$. Also note that the codimension of $Z_{i, j}$ for $d$ coincides with the codimension of $Z_{1, j-i+1}$ for $e$, the first variety is just a product of the latter with an affine space. Consequently, $Z_{i, j}$ for $d$ is irreducible precisely when $Z_{1, j-i+1}$ is irreducible for $e$. Finally, we compare the component $\mathfrak{n}(T(i, j))$ for $d$ with the unique component $\mathfrak{n}(T(1, j-i+1))$ for $e$ that coincides with $\mathfrak{n} \cap C(\mu(1, j-i+1))$ for $e$. Again, both are just given by the equation $\operatorname{rk} A[i, j]^{\kappa(i, j)}<r_{i, j}^{\kappa(i, j)}$ for $d$, respectively, rk $A[1, j-i+1]^{\kappa(1, j-i+1)}<r_{1, j-i+1}^{\kappa(1, j-i+1)}$ for $e$. This finally shows that both varieties coincide.

\section{The irreducible components of $Z$}

We are now ready to finish the proof of the descriptions of the decomposition of the complement $Z=\mathfrak{n} \backslash \mathcal{O}(d)$ of the Richardson orbit into irreducible components. Again, let $d=$ $\left(d_{1}, \ldots, d_{t}\right)$ be a dimension vector, $\lambda=\lambda(d)$ the partition of the Richardson orbit and $(i, j)$ a pair with $1 \leq i<j \leq t$. Recall that the $T(i, j)$ are elements of $\mathcal{T}(\mu(i, j), d)$. By Proposition 3.3 the $T(i, j)$ correspond to irreducible components of $\mathfrak{n} \cap C(\mu(i, j))$. So the corresponding $\mathfrak{n}(T(i, j))$ are irreducible.

\section{Theorem 4.1}

$$
Z=\bigcup_{(i, j) \in \Lambda(d)} Z_{i j}
$$

is the decomposition of $Z$ into irreducible components.

Proof We know that $Z$ is the union of all $Z_{i j}$ over all $(i, j)$ with $1 \leq i<j \leq t$ from Lemma 2.10. By Lemma 2.11,

$$
Z=\bigcup_{(k, l) \in \Gamma^{\prime}(d)} Z_{k l}
$$

for some subset $\Gamma^{\prime}(d) \subseteq \Gamma(d)$. And finally, Corollary 2.13 tells us that for each $(k, l)$ in this subset $\Gamma^{\prime}(d)$, there exists $(i, j) \in \Lambda(d)$ such that $Z_{k l}$ is contained in $Z_{i j}$.

It remains to see that $Z_{i j} \subsetneq Z_{k l}$ and $Z_{i j} \supsetneq Z_{k l}$ for all $(i, j) \neq(k, l) \in \Lambda(d)$. This follows as for $(i, j) \neq(k, l)$ from $\Lambda(d)$, one can find matrices $A$ in $Z_{i j}$ which do not satisfy the conditions for $Z_{k l}$ and vice versa: assume $(i, j) \neq(k, l) \in \Lambda(d)$. From the line diagram $L_{R}(d)$ we remove one edge of the lowest chain connecting columns $i$ and $j$, connecting the resulting edges if possible with lower rows to the left and right (as with the dashed lines in 
Fig. 5) produces an element $A$ of $Z_{i j}$ (under $\Phi$ ) with $A \notin Z_{k l}$. It is completely analogous to find $B \in Z_{k l}, B \notin Z_{i j}$ The irreducibility follows now since $Z_{i j}=\mathfrak{n}(T(i, j))$ (Lemma 3.9).

Corollary 4.2 The complement $Z=\mathfrak{n} \backslash \mathcal{O}(d)$ has at most $t-1$ irreducible components.

Proof If $d$ is increasing or decreasing then clearly, $\Lambda(d)$ has size $t-1$, cf. Example 1.2. The same is true if the $d_{i}$ are all different. In all other cases there are $d_{i}=d_{j}$ with $|j-i|>1$, and such that there exists an index $i<l<j$ with $d_{l} \neq d_{i}$. If $d_{l}>d_{i}$ is minimal among these, then neither $(i, l)$ nor $(l, j)$ belong to $\Lambda(d)$ and thus $\Lambda(d)$ has at most $t-2$ elements. The same is true for $d_{l}<d_{i}, d_{l}$ maximal among such.

Furthermore, we can describe the codimension of $Z_{i j}$ in $\mathfrak{n}$ as follows. Recall that $T(i, j)$ is obtained from $T(d)$ through a minimal movement (see Sect. 3.1). Let $c(i, j)$ be the number of rows the box with label $j$ moves down, i.e. $j$ goes from row $s(i, j)$ to row $s(i, j)+c(i, j)$. It is known that for every row a box in a Young diagram is moved down, the dimension of the $\mathrm{GL}_{n}$-orbit of the corresponding nilpotent elements decreases by two. This can be seen using the formula for the dimension of the stabilizer from [11]. The change in dimension in the nilradical is half of this. Thus, the resulting $\mathfrak{n}(T(i, j))$ then has codimension $c(i, j)$ in the nilradical $\mathfrak{n}$ and we get:

Corollary 4.3 For $(i, j) \in \Gamma(d), Z_{i j}$ has codimension $c(i, j)$ in $\mathfrak{n}$.

The second description of the irreducible components of $Z$ is now an immediate consequence of Theorem 4.1 and Lemma 3.9:

\section{Corollary 4.4}

$$
Z=\bigcup_{(i, j) \in \Lambda(d)} \mathfrak{n}(T(i, j))
$$

is the decomposition of $Z$ into irreducible components.

\section{An application}

In the last section, we illustrate our work on an example. We work with $G=\mathrm{GL}_{5}$ and consider the parabolic subgroups of different dimension vectors.

(A) If $d=(1,1,1,1,1)$ then $P=B$ is a Borel subgroup. Note that $\Lambda(d)=\Gamma(d)=$ $\{(1,2),(2,3),(3,4),(4,5)\}$, so Theorem 4.1 describes the complement $Z$ as the union

$$
Z=Z_{12} \cup Z_{23} \cup Z_{34} \cup Z_{45}
$$

of four irreducible components.

In this example, we have that $A_{i j}=a_{i j}$ are all $1 \times 1$-matrices. The Richardson orbit is the intersection of the regular nilpotent orbit with the set of upper triangular matrices in $\mathfrak{g l}_{5}$. The regular nilpotent elements are the nilpotent $5 \times 5$-matrices whose fourth power is non-zero. So the Richardson orbit consists of the strictly upper triangular matrices $A=\left(a_{i j}\right)_{i j}$ with 


$$
A[1,5]^{4}=\left(\begin{array}{ccccc}
0 & 0 & 0 & 0 & a_{12} a_{23} a_{34} a_{45} \\
& 0 & 0 & 0 & 0 \\
& & 0 & 0 & 0 \\
& & & 0 & 0 \\
& & & & 0
\end{array}\right) \quad \text { with } a_{12} a_{23} a_{34} a_{45} \neq 0
$$

For $A$ to be in the complement $Z$ of the Richardson orbit, the product $a_{12} a_{23} a_{34} a_{45}$ has to be zero, i.e. $A[1,5]^{4}=0$. Then clearly, $A \in Z_{i, i+1}$ for an $i \leq 4$ as $Z_{i, i+1}$ is the set of matrices with $A_{i, i+1}=0$. Thus, $A$ lies in one of the components $Z_{i j}$ with $(i, j) \in \Lambda(d)$.

(B) If $d=(1,1,1,2)$ then $\Lambda(d)=\Gamma(d)=\{(1,2),(2,3),(3,4)\}$. The Richardson orbit is determined by the conditions $\operatorname{rk} A[12]=\operatorname{rk} A[23]=\operatorname{rk} A[34]=1, \operatorname{rk} A[13]^{2}=$ rk $A[24]^{2}=1, \operatorname{rk} A[14]^{3}=1$ (for $A \in \mathfrak{n}$ ). For $A$ to be in the complement, one of these ranks has to be zero. By Theorem 4.1, we should have

$$
Z=Z_{12} \cup Z_{23} \cup Z_{34}
$$

where the component $Z_{12}$ consists of the matrices $A \in \mathfrak{n}$ with $a_{12}=0$, the component $Z_{23}$ of the $A$ with $a_{23}=0$ and $Z_{34}$ of the $A$ with $a_{34}=a_{35}=0$. Let us first compute $A^{2}$, and $A^{3}$ for $A \in \mathfrak{n}$ (we omit the zero entries in the opposite nilradical):

$$
\begin{aligned}
& A=\left(\begin{array}{ccccc}
0 & a_{12} & a_{13} & a_{14} & a_{15} \\
& 0 & a_{23} & a_{24} & a_{25} \\
& & 0 & a_{34} & a_{35} \\
& & & 0 & 0 \\
& & & & 0
\end{array}\right) \\
& A^{2}=\left(\begin{array}{ccccc}
0 & 0 & a_{12} a_{23} & a_{12} a_{24}+a_{13} a_{34} & a_{12} a_{25}+a_{13} a_{35} \\
& 0 & 0 & a_{23} a_{34} & a_{23} a_{35} \\
v & & 0 & 0 & 0 \\
& & & 0 & 0 \\
& & & & 0
\end{array}\right) \\
& A^{3}=\left(\begin{array}{ccccc}
0 & 0 & 0 & a_{12} a_{23} a_{34} & a_{12} a_{23} a_{35} \\
& 0 & 0 & 0 & 0 \\
v & & 0 & 0 & 0 \\
& & & 0 & 0 \\
& & & & 0
\end{array}\right)
\end{aligned}
$$

Then we see that $A[14]^{3}=A^{3}=0$ if and only if $a_{12} a_{23} a_{34}=0$ and $a_{12} a_{23} a_{35}=0$. Thus, $A$ clearly belongs to one of the three components described above. Now, $A[13]^{2}=0$ if and only if $a_{12} a_{23}=0$ as this is the only non-zero entry of $A[13]^{2}$. Similarly, $A[24]^{2}=0$ if and only if $a_{23} a_{34}=0$ and $a_{23} a_{35}=0$. In all cases, $A$ is contained in one of the three components. The case of $d=(2,1,1,1)$ is completely analogous.

(C) The first interesting case appears for $d=(1,1,2,1)$. Here, $\Lambda(d)=\{(1,2),(2,4)\} \neq$ $\Gamma(d)$. So we expect two irreducible components, $Z_{12}$ as the matrices $A$ with $a_{12}=0$ 
and $Z_{24}$ as the $A$ with rk $A[24]^{2}=0$. We first compute $A, A^{2}$ and $A^{3}$ for $A \in \mathfrak{n}$ :

$$
\begin{aligned}
& A=\left(\begin{array}{ccccc}
0 & a_{12} & a_{13} & a_{14} & a_{15} \\
& 0 & a_{23} & a_{24} & a_{25} \\
& & 0 & 0 & a_{35} \\
& & & 0 & a_{45} \\
& & & & 0
\end{array}\right) \\
& A^{2}=\left(\begin{array}{ccccc}
0 & 0 & a_{12} a_{23} & a_{12} a_{24} & a_{12} a_{25}+a_{13} a_{35}+a_{14} a_{45} \\
& 0 & 0 & a_{23} a_{35} & a_{24} a_{45} \\
& & 0 & 0 & 0 \\
& & & 0 & 0 \\
& & & & 0
\end{array}\right) \\
& A^{3}=\left(\begin{array}{ccccc}
0 & 0 & 0 & 0 & a_{12}\left(a_{23} a_{34}+a_{24} a_{45}\right) \\
& 0 & 0 & 0 & 0 \\
& & 0 & 0 & 0 \\
& & & 0 & 0 \\
& & & & 0
\end{array}\right)
\end{aligned}
$$

The elements $A$ of the Richardson orbit have non-zero $a_{12}$, and $\operatorname{rk} A[23]=\mathrm{rk}$ $A[34]=1, \operatorname{rk} A[13]^{2}=\operatorname{rk} A[24]^{2}=\operatorname{rk} A[14]^{3}=\operatorname{rk} A^{3}=1$. Clearly, when $a_{12}=0$, then $A \in Z_{12}$. And when rk $A[23]$ rk $A[34]=0, A$ belongs to $Z_{24}$. Now $A[14]^{3}=0$ if and only if $a_{12}=0$ or $a_{23} a_{34}+a_{24} a_{34}=0$ which is equivalent to $A \in Z_{12}$ or $A \in Z_{24}$, respectively. Furthermore, $A[13]^{2}=0$ if and only if $a_{12} a_{23}=0$ and $a_{12} a_{24}=0$, which is equivalent to $A \in Z_{12} \cup Z_{24}$. The matrices $A$ satisfying $A[24]^{2}$ are by definition $Z_{24}$. The case $d=(1,2,1,1)$ is analogous.

(D) Let $d=(2,2,1)$, with $\Lambda(d)=\Gamma(d)=\{(1,2),(2,3)\}$, the complement should be $Z_{12} \cup Z_{23}$. The Richardson orbit is given as the matrices $A$ with rk $A[12]=2$ and rk $A[23]=\operatorname{rk} A[13]^{2}=1$. We compute $A$ and $A^{2}$ :

$$
A=\left(\begin{array}{ccccc}
0 & 0 & a_{13} & a_{14} & a_{15} \\
& 0 & a_{23} & a_{24} & a_{25} \\
& & 0 & 0 & a_{35} \\
& & & 0 & a_{45} \\
& & & & 0
\end{array}\right) \quad A^{2}=\left(\begin{array}{ccccc}
0 & 0 & 0 & 0 & a_{13} a_{35}+a_{14} a_{45} \\
& 0 & 0 & 0 & a_{23} a_{35}+a_{24} a_{45} \\
& & 0 & 0 & 0 \\
& & & 0 & 0 \\
& & & & 0
\end{array}\right)
$$

If $A$ is a matrix with $A[13]^{2}=0$ then if $a_{35}=a_{45}=0, A$ is an element of $Z_{23}$. So let rk $A[23] \neq 0$. Solving the two equations $a_{13} a_{35}+a_{14} a_{45}=0 a_{23} a_{35}+a_{24} a_{45}=0$ then shows that the rank of $A[12]$ is one. Thus $Z_{13}$ is already contained in $Z_{12}$.

The case $d=(1,2,2)$ is analogous.

(E) The second interesting case is $d=(2,1,2)$, with $\Lambda(d)=\{(1,3)\}$ and $\Gamma(d)=$ $\{(1,3),(1,2),(2,3)\}$. Here we only obtain one irreducible component in the complement! The Richardson orbit is defined by $\mathrm{rk} A[13]^{2}=\mathrm{rk} A^{2}=1$ and $\mathrm{rk} A=3$ : the dimension of its stabilizer has to be equal to the dimension of the Levi factor. Using the formulae from [11] then gives this description of the Richardson orbit. For the complement, we are looking at matrices $A$ with $\operatorname{rk} A[12]=0$ or $\operatorname{rk} A[23]=0$ or rk $A[13]^{2}=0$. If $A$ satisfies $A[12]=0$ then $A^{2}$ is also zero, so $A \in Z_{13}$ by definition. Similarly, matrices with $A[23]=0$ square to zero and hence lie in $Z_{13}$.

(F) The case $d=(1,3,1)$ with $\Lambda(d)=\{(1,3)\}$, so again, we only have one component in the complement of the open dense orbit. For matrices of the Richardson orbit, we have $\operatorname{rk} A[12]=\operatorname{rk} A[23]=\operatorname{rk} A[13]^{2}=1$. For the complement, we take matrices 
where one of these ranks is zero. If it is rk $A[12]=0$ or rk $A[23]=0$ then clearly, $A[13]^{2}=0$, so $A \in Z_{13}$. The cases $d=(3,1,1)$ and $d=(1,1,3)$ behave similarly as $d=(2,1,1,1)$ and $d=(1,1,1,2)$. We omit them here.

(G) The remaining cases are $d=(4,1), d=(1,4)$. Here, the complement to the Richardson orbit is given by $A[12]=0$, i.e. it is the zero matrix.

\section{References}

1. Baur, K.: Richardson elements for classical Lie algebras. J. Algebra 297(1), 168-185 (2006)

2. Baur, K., Hille, L.: On the complement of the dense orbit for a quiver of type $\mathbb{A}$. Preprint

3. Brüstle, T., Hille, L.: Finite, tame, and wild actions of parabolic subgroups in $G L(V)$ on certain unipotent subgroups. J. Algebra 226(1), 347-360 (2000)

4. Brüstle, T., Hille, L.: Matrices over upper triangular bimodules and $\Delta$-filtered modules over quasihereditary algebras. Colloq. Math. 83(2), 295-303 (2000)

5. Brüstle, T., Hille, L.: Actions of parabolic subgroups in $\mathrm{GL}_{n}$ on unipotent normal subgroups and quasihereditary algebras. Colloq. Math. 83(2), 281-294 (2000)

6. Brüstle, T., Hille, L., Ringel, C., Röhrle, G.: The $\Delta$-filtered modules without self-extensions for the Auslander algebra of $k[T] /\langle T n\rangle$. Algebr. Represent. Theory 2(3), 295-312 (1999)

7. Brüstle, T., Hille, L., Röhrle, G.: Finiteness for parabolic group actions in classical groups. Arch. Math. (Basel) 76(2), 81-87 (2001)

8. Fulton, W.: Young Tableaux. Cambridge University Press, London (1997)

9. Goodwin, S.M., Hille, L., Röhrle, G.: The orbit structure of Dynkin curves. Math. Z. 257(2), 439-451 (2007)

10. Hille, L.: Aktionen algebraischer Gruppen, geometrische Quotienten und Köcher. Habilitationsschrift, Hamburg (2003)

11. Kraft, H., Procesi, C.: On the geometry of conjugacy classes in classical groups. Comment. Math. Helv. 57(4), 539-602 (1982)

12. Hille, L., Röhrle, G.: A classification of parabolic subgroups of classical groups with a finite number of orbits on the unipotent radical. Transform. Groups 4(1), 3552 (1999)

13. Richardson, R.W.: Conjugacy classes in parabolic subgroups of semisimple algebraic groups. Bull. Lond. Math. Soc. 6, 21-24 (1974)

14. Spaltenstein, N.: Classes Unipotentes et Dous-Groupes de Borel. Lecture Notes in Mathematics, vol. 946. Springer, Berlin (1982) 